\title{
Network-Assisted Optimal Datalink Selection Scheme for Heterogeneous Aeronautical Network
}

\author{
Dongli Wang $\mathbb{C}^{1},{ }^{1}$ Guoce Huang, ${ }^{2}$ Shufu Dong, ${ }^{2}$ Yequn Wang, ${ }^{2}$ Jian Liu, ${ }^{2}$ and Weiting Gao ${ }^{2}$ \\ ${ }^{1}$ Graduate College, Air Force Engineering University, Xian 710051, China \\ ${ }^{2}$ Information and Navigation College, Air Force Engineering University, Xian 710077, China \\ Correspondence should be addressed to Dongli Wang; wd14178@163.com
}

Received 23 July 2018; Revised 9 September 2018; Accepted 13 September 2018; Published 16 October 2018

Guest Editor: Phuc V. Trinh

Copyright (c) 2018 Dongli Wang et al. This is an open access article distributed under the Creative Commons Attribution License, which permits unrestricted use, distribution, and reproduction in any medium, provided the original work is properly cited.

\begin{abstract}
Currently, the existing datalink selection algorithms in heterogeneous aeronautical network unilaterally focus on the user side or network side and lack the two sides' cooperation, which seriously affects the utilization of available datalinks. Firstly, to reflect the user-side requirements and network-side load, we propose a user-centric datalink selection scheme combing the user-side multiattribute utility and the network-side access rate for single-user selection. It can be established as a multiobjective optimization problem and solved by the selected optimal datalink. Secondly, for multiuser datalink selection, in order to guarantee the access requests of multiple users, the central control mode of Software Define Networking (SDN) is used to assist the user to perform datalink selection on the network side. By constructing multiuser multiobjective optimization problem, a priority distinction selection (PDS) algorithm is proposed to maximize the number of users accessing their optimal datalinks and minimize the modification of the users' access request. Finally, the proposed user-side datalink selection scheme and network-side assisted selection algorithm are simulated to verify the feasibility and effectiveness.
\end{abstract}

\section{Introduction}

In the field of aeronautical communications, with the anticipated increase in the number of aircraft [1], the communication demands have shown exponential growth in numerous applications, such as air traffic control (ATC) for manned aircraft [2], communication and surveillance for unmanned aerial vehicles (UAVs) [3-5], and the cooperative systems of manned-unmanned aerial vehicles [6]. In all of these applications, a great mass of heterogeneous information with different criticality levels needs to be transmitted over the datalinks. However, it is impractical to use a single datalink to meet all requirements in air-to-air and air-to-ground communications [7]. Different datalinks have obvious differences in their technical indicators, usage costs, and potential risks and are applicable to diverse scenarios. Due to the diversity of aircraft and the specificity of the terrestrial networks corresponding to different links, the aeronautical network composed of aircraft, transmission links, and terrestrial access networks exhibits apparent heterogeneity, which is more complexity than the homogeneous network. However, the convergence of multiple heterogeneous transmission links can extend system capacity and utilize heterogeneous resources efficiently, which is unrealizable for a single link. Therefore, by integrating multiple Radio Access Technologies (RATs), Next Generation Air Transportation System (NextGen) [8] and Signal European Sky for ATM Research (SESAR) [9] conduct researches on heterogeneous aeronautical communication systems including NEWSKY (NEtWorking the SKY) [10] and SANDRA (Seamless Aeronautical Networking through integration of Data links, Radios, and Antennas) [11], which have achieved significant progress. The heterogeneous aeronautical communication system constructs the network architecture by the airborne segment, the link segment and the ground segment. It introduces the Multilink Operational Concept (MLOC) on the link segment and isolates the applications from different RATs on the airborne segment. Combined with the construction of the heterogeneous aeronautical network on the ground segment, it is possible to integrate the future datalinks such as L-band 
Digital Aeronautical Communications System (LDACS) [12] and Aeronautical Mobile Airport Communications System (AeroMACS) [13] and to be compatible with the original technologies, such as very high frequency (VHF) digital link mode 2 (VDL 2) [14].

The aircraft generally has multilink configuration and multihoming capabilities to ensure flight safety and reliability of information transmission. In the scenario where multiple links are available, suitable datalink selection is needed to maintain seamless communications [15]. As we know, there are four main reasons for triggering the aircraft to select datalink [16]: (1) changes in the flight phase of the aircraft lead to changes in available links, (2) arrival of new or handover services, (3) dynamic changes of user-side attributes or network-side properties, and (4) changes in user or operator preferences. The usual datalink selection algorithm is based on a preset priority. For example, the VHF link has the highest priority, followed by the satellite link and High Frequency (HF) link. This method does not consider the attributes of the services and easily causes the network congestion, long access time, and unguaranteed communication effect. In addition, the baseline link selection algorithm used in SANDRA is also one of the commonly used methods [17]. It selects available links according to a single attribute. This algorithm is too simple to consider user preference and multiple attributes, so the selected datalink is not optimal. Compared with single-attribute link selection, the multiattribute link selection method considers multiple factors and has a better user experience. The optimal datalink selection algorithm proposed by ALAM et al. utilizes the multiple attribute decision making (MADM) method to consider user preference, and has remarkable flexibility [17].

The existing datalink selection algorithms in heterogeneous aeronautical network unilaterally focus on the user side or network side, and usually use user-side attributes and preferences as the basis for datalink selection instead of network-side dynamic attributes. Considering the wide coverage of aeronautical links, such as the Iridium beam footprint with a diameter of approximately 550 kilometres [18] and the maximum transmission range of 200 nautical miles of LDACS, it is more than one aircraft in the overlay airspace as shown in Figure 1. Simultaneously, multiple aircraft are likely to perform datalink selection due to their high-speed mobility. If plenty of aircraft select the same datalink, the access network will be congested with excessive load. So it is necessary to estimate the network-side load to guide the link selection on the user side. Moreover, according to the limited resources of the heterogeneous network and the different available datalinks of each user, we should consider that how to maximize the protection of the userside access requests with multiuser access and network-side assistance.

In this paper, we propose a network-assisted optimal datalink selection scheme for the heterogeneous aeronautical network. Firstly, for single-user selection a multiattribute utility function is constructed according to the user-side requirements, and the access rate is measured to present the network-side load. The multiobjective optimization problem is implemented to reflect the user-side demand and the

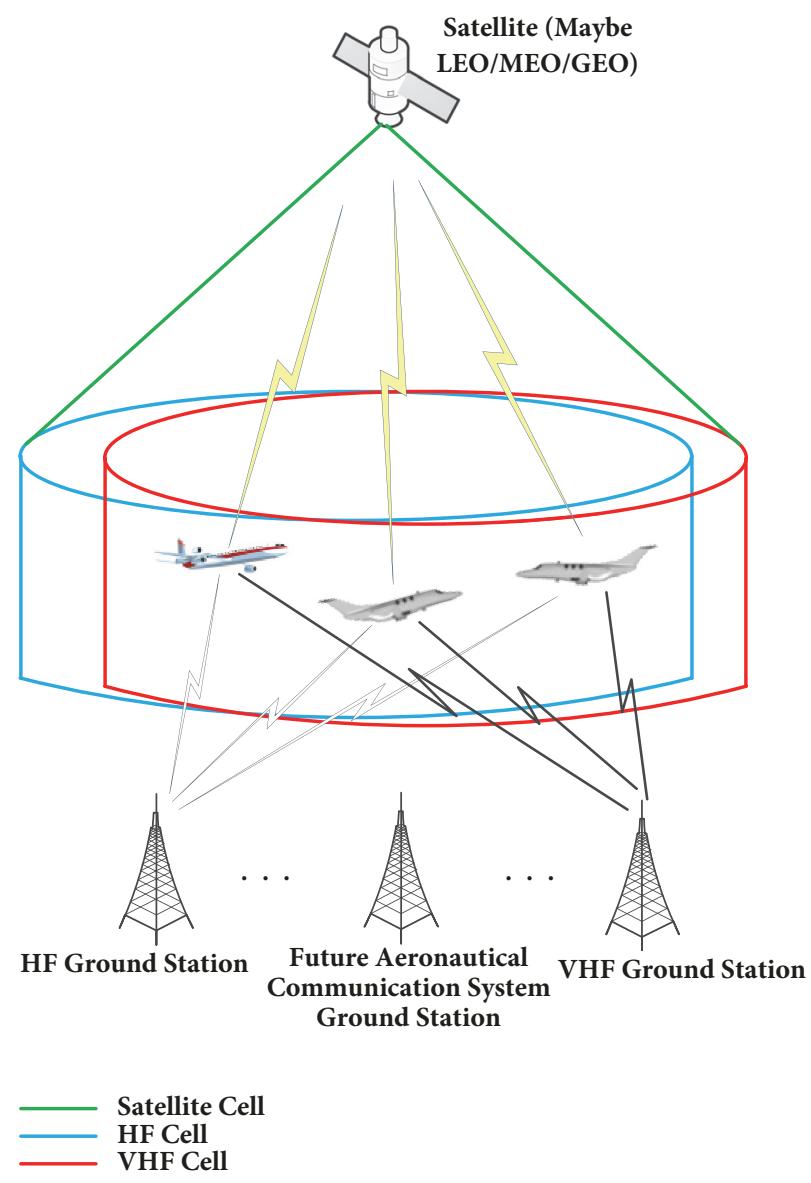

FIGURE 1: Multilink overlapping airspace scenario.

network-side dynamic, and then converted to a singleobjective optimization problem solved by the selected optimal link. Secondly, for multiuser link selection, in order to guarantee the access requests of multiple users, the central control mode of Software Define Networking (SDN) [19] is used to assist the user to perform link selection on the network side. By constructing multiuser multiobjective optimization problem, a priority distinction selection algorithm is proposed to maximize the number of users accessing their optimal datalinks and minimize the modification of the user's access request. Finally, the proposed user-side datalink selection scheme and network-side assisted selection algorithm are simulated to verify the feasibility and effectiveness.

This paper is organized as follows. Section 2 establishes the system model and proposes the evaluation models on user side and network side, respectively. The novel datalink selection scheme assisted by the centric network architecture is presented in Section 3. Simulation results are presented in Section 4 and Section 5 concludes the paper.

\section{System Description and Problem Formulation}

In this section, we formulate the datalink selection problem in the heterogeneous aeronautical network. Specifically, we 


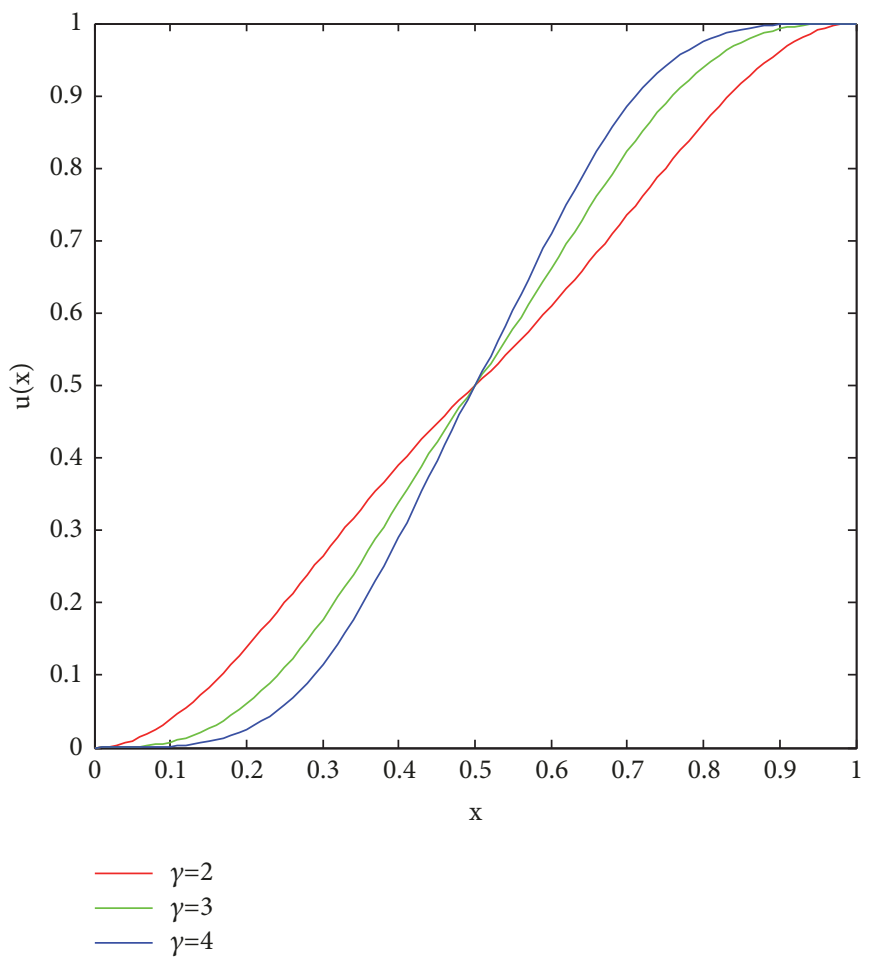

FIGURE 2: The utility function under different $\gamma$.

consider a heterogeneous aeronautical environment which consists of $\mathrm{M}$ different datalinks and $\mathrm{N}$ aircraft in the overlapping airspace, as shown in Figure 1. For each datalink, it corresponds to one different ground access network. We assume that every aircraft is independent with each other, and the scene of cooperation between different aircraft is not considered in this paper. Note that network selection and link selection have the same meaning because the end point of the link is the access network, and the terms users and aircraft are interchangeable in this paper.

2.1. The Utility Function on User Side. To effectively evaluate the different attributes of datalinks, we design a universal utility function on user side to map different attributes to corresponding utility metrics. For the sake of generality, we use the Sigmoid function to build this utility that satisfies the following additional conditions:

$$
\begin{aligned}
& u(x)=0 \quad \forall x \leq x_{\min } \\
& u(x)=1 \quad \forall x \geq x_{\max } \\
& u(x)=0.5, \quad x=x_{\operatorname{mid}}
\end{aligned}
$$

where $x_{\max }$ and $x_{\min }$ are the maximum and minimum values of the attribute $x$, respectively, and $x_{\operatorname{mid}}=\left(x_{\max }+x_{\min }\right) / 2$ is the median value.

We construct the utility function that meets (1) as follows [20]:

$$
\begin{aligned}
& u(x) \\
& = \begin{cases}0 & x \leq x_{\min } \\
\frac{\left(x / x_{\operatorname{mid}}\right)^{\gamma}}{1+\left(x / x_{\operatorname{mid}}\right)^{\gamma}} & x_{\min }<x \leq x_{\operatorname{mid}} \\
1-\frac{\left(\left(x_{\max }-x\right) /\left(x_{\max }-x_{\operatorname{mid}}\right)\right)^{\gamma}}{1+\left(\left(x_{\max }-x\right) /\left(x_{\max }-x_{\text {mid }}\right)\right)^{\gamma}} & x_{\operatorname{mid}}<x \leq x_{\max } \\
1 & x>x_{\max }\end{cases}
\end{aligned}
$$

where $\gamma \geq 2$. Figure 2 shows the utility function of different $\gamma$. It is straightforward that this utility function is twice differentiable, monotonic, and concavity-convex and satisfies the utility theory [20].

Generally, the attributes of each different link are classified into upward attributes and downward attributes according to their anticipated values. For upward attributes, such as received signal strength (RSS) and transmission rate, we directly use $u(x)$ as their utility function. On the contrary, $1-u(x)$ is adopted as the utility function for downward attributes, e.g., cost, bit error rate, and delay.

In order to evaluate the datalink more comprehensively, it is not enough to only consider a single attribute, so an overall utility combined by multiple attributes is inevitable. The combined multiattribute utility function should follow the following criteria:

$$
\begin{gathered}
\frac{\partial U(\mathbf{x})}{\partial u_{i}} \geq 0 \\
\operatorname{sign}\left(\frac{\partial U(\mathbf{x})}{\partial x_{i}}\right)=\operatorname{sign}\left(\frac{d u_{i}}{d x_{i}}\right)
\end{gathered}
$$




$$
\begin{aligned}
\lim _{u_{i} \longrightarrow 0} U(\mathbf{x}) & =0, \quad \forall i=1, \ldots n \\
\lim _{u_{1}, \ldots, u_{n} \rightarrow 1} U(\mathbf{x}) & =1
\end{aligned}
$$

In the multiattribute utility function, the exponentialmultiplication form is commonly used with satisfying constraints (3) [20]. Thus the overall utility of the user $j$ corresponding to the link $e_{i}$ can be constructed as

$$
U_{i j}(\mathbf{x})=\prod_{k=1}^{K} u_{i k}\left(x_{i k}\right)^{\omega_{k}}, \quad i=1,2, \ldots, M
$$

where $\mathbf{x}$ is the vector consisting of $x_{i k} ; \omega_{k} \in[0,1]$ is the weight of the $k$ th attribute $x_{i k}$ of the link $e_{i}$ and the utility function $u_{i k}\left(x_{i k}\right)$. It is apparent that $\sum_{k=1}^{K} \omega_{k}=1$, where $K$ is the number of attributes. Although different services of aircraft have different requirements on the importance of each attribute, the weight of each attribute for the same service is fixed for different links and the i-TRUST method can be adopted to determine its value [17].

2.2. The Access Rate on Network Side. Given that there is more than one aircraft that triggers link selection in the overlapping airspace, the load of the access network connected by different link has to be considered to avoid access failure caused by multiuser access simultaneously. However, the increasing flight security problem reminds aircraft do not share their private information with others in a noncooperative scenario. The current selecting user has no idea of the number of other users selecting the same link, and does not know the resources they requested, so it is a challenging problem for current selecting user to select the optimal link that satisfies its required resources and maximize the access rate of target network. Thus, we consider the access rate, which means the probability of current selecting user successfully accessing the target link, as the evaluation metric on the network side.

On account of the imperfect network information about other selecting users, the current selecting user has to estimate this information by the existing knowledge and then regard it as the evaluation criterion on the network side when performing the datalink selection. Therefore, in order to assist a selecting user to infer the link selection behaviours of other selecting users, we investigate the relationship between any two selecting users based on their available link sets, because each one has several available links in the overlapping airspace. According to [21], we use the intersection of available link sets of the two users as the criterion. When the intersection is empty, the two are completely uncorrelated, which means the correlation coefficient is 0 and their link selection behaviours are totally unaffected. Conversely, when the available link sets of the two are identical, the two are absolutely related and their correlation coefficient is 1 , which causes that the link selection behaviours have the greatest impacts on accessing the target network. If the intersection is a part of the available link set, then the two will be partially related and the correlation coefficient is between 0 and 1 . So in order to estimate the access rate of the target network, the probability of other users selection target network and their requirements on link resources are two prerequisites.

According to the above analysis, considering the worst case, it is assumed that all selecting users are completely related to the current selecting user, which has the greatest influence on the user link selection. In the heterogeneous aeronautical network, we assume that the current number of users and the remaining available resources of each access network are embedded in the air intelligence, and are periodically transmitted by broadcast to each user in the current airspace. It can be seen that the number of users in the current airspace is $N=\sum_{i=1}^{M} N_{i}$, where $N_{i}$ is the number of users accessing the network $e_{i}$. Since the resources used by different multiple access methods are generally different, such as the channel of Frequency Division Multiple Access (FDMA), time slot of Time Division Multiple Access (TDMA), code word of Code Division Multiple Access (CDMA) and timefrequency resource block of Orthogonal Frequency Division Multiple Access (OFDMA), a unified measurement criterion using bit rate is reasonable for different access networks. Therefore, the remaining available bit rate of the current network can be adopted to measure its available resources uniformly, which can be expressed as

$$
R_{s}^{i}=\left(N_{\max }^{i}-N_{i}\right) R_{\max }^{i}
$$

where $N_{\max }^{i}$ is the maximum number of users supported by the link $e_{i}$ in the current airspace and $R_{\max }^{i}$ is the maximum bit rate supported by the link $e_{i}$.

To ensure the current selecting user to successfully access to the network connected by target link, two factors need to be met. Firstly, the current user needs to select the expected link as the target link. Then, the number of users selecting the target link should be smaller than the maximum number of users supported by the target link in the current airspace, which means that the target link is not fully loaded. Since the target link is selected on the user side according to the overall utility of each link, we can calculate the selected probability of the target link among $\mathrm{M}$ datalinks, and its probability can be given by

$$
\alpha_{i j}=\frac{U_{i j}(\mathbf{x})}{\sum_{i=1}^{M} U_{i j}(\mathbf{x})}
$$

Secondly, the underload probability of the corresponding target network is calculated. The current selecting user lacks of the information of other selecting users in the same airspace, so we assume that other selecting users can select the best link based on available resources according to the Always Best Connection (ABC) [22] criterion. The probability of each user selecting a link based on available resources can be expressed as

$$
q_{i}=\frac{R_{s}^{i}}{\sum_{i=1}^{M} R_{s}^{i}}
$$

For the target link, the total number of users at the next moment consists of two parts: one is the number of users switching from other links to the current link $N_{o 2 i}$; the other 
is the number of users who maintain the access to current link $N_{i 2 i}$. The probability of the former $N_{o 2 i}$ can be calculated by the current selecting user according to the estimated information of other selecting users as follows:

$$
\begin{aligned}
P & \left\{N_{o 2 i} \mid N-N_{i}-1\right\} \\
& =\left(\begin{array}{c}
N-N_{i}-1 \\
N_{o 2 i}
\end{array}\right)\left(q_{i}\right)^{N_{o 2 i}}\left(1-q_{i}\right)^{N-N_{i}-1-N_{o 2 i}}
\end{aligned}
$$

Following the same reason, the distribution probability of the latter $N_{i 2 i}$ can be given by

$$
P\left\{N_{i 2 i} \mid N_{i}\right\}=\left(\begin{array}{c}
N_{i} \\
N_{i 2 i}
\end{array}\right)\left(\gamma_{i}\right)^{N_{i 2 i}}\left(1-\gamma_{i}\right)^{N_{i}-N_{i 2 i}}
$$

where $\gamma_{i}$ is the probability of user staying on the current link and can be estimated by the same probability of current selecting user.

Only when $N_{i 2 i}+N_{o 2 i} \leq N_{\max }^{i}-1$ does the current selecting user accessing the target link have no collisions with other users, therefore, the underload probability of target link is expressed as

$$
P_{\text {Underload }}^{i}=P\left\{N_{i 2 i}+N_{o 2 i} \leq N_{\text {max }}^{i}-1\right\}
$$

where $N_{i 2 i}+N_{o 2 i}$ is a simple two-dimensional random variable.

Consequently, the access rate of the target network can be obtained as follows:

$$
P_{\text {Access }}^{i j}=\alpha_{i j} P_{\text {Underload }}^{i}
$$

\section{The Proposed Datalink Selection Scheme}

In this section, we propose the datalink selection on the user side and the network-assisted selection scheme on the network side.

3.1. User-Side Selection Scheme. Since the users generally have a multilink configuration for safety, we only consider the scenario of selecting the optimal link when multiple links are available and use $\beta_{i j}$ to indicate the result of link selection, where

$$
\beta_{i j}= \begin{cases}1, & \text { if current user select datalink } e_{i} \\ 0, & \text { otherwise }\end{cases}
$$

When only the link selection on the user side is considered, the user desires to select the link with the largest overall utility. At this time, the user-side evaluation model can be established as the following optimization problem:

$$
\begin{array}{ll}
\max & \mathcal{O}_{j}^{1}=\sum_{i=1}^{M} \beta_{i j} U_{i j}(\mathbf{x}) \\
\text { s.t. } & \sum_{i=1}^{M} \beta_{i j} \leq 1
\end{array}
$$

where $\sum_{i=1}^{M} \beta_{i j} \leq 1$ means that at most one link can be selected among all the available links, and the constraint can be easily extended to the selection of multiple links.
Similarly, when only considering the link selection on the network side, the user expects to select the link with the highest access rate. The optimization problem established by using the network-side evaluation model is given by

$$
\begin{array}{ll}
\max & \mathcal{O}_{j}^{2}=\sum_{i=1}^{M} \beta_{i j} P_{\text {Access }}^{i j} \\
\text { s.t. } & \sum_{i=1}^{M} \beta_{i j} \leq 1
\end{array}
$$

However, in the actual link selection, the user should not only consider the user-side evaluation model, but also consider the evaluation model on the network side; that means the current selecting user expects to both obtain the maximum utility and the highest access rate. Consequently, combining $\mathcal{O}_{j}^{1}$ and $\mathcal{O}_{j}^{2}$ under the same constraints, the multiobjective optimization problem $\left\{\mathcal{O}_{j}^{1}, \mathcal{O}_{j}^{2}\right\}$ can be established. For the multiobjective optimization, according to the importance of each target, the simple weighting method is usually adopted to transform multiple objectives into a single-objective optimization problem to obtain an acceptable solution under certain compromise [23]. According to [24], the feasible solution of transformed single-objective optimization is one of Pareto optimal solutions when each weight of single target is greater than zero.

Therefore, the multiobjective optimization problem $\left\{\mathcal{O}_{j}^{1}, \mathcal{O}_{j}^{2}\right\}$ under the same constraints of $\mathcal{O}_{j}^{1}$ or $\mathcal{O}_{j}^{2}$ can be transformed into the following single-objective optimization problem:

$$
\begin{aligned}
\max & \mathcal{O}_{j}=\lambda_{j} \cdot \mathcal{O}_{j}^{1}+\left(1-\lambda_{j}\right) \cdot \mathcal{O}_{j}^{2} \\
\text { s.t. } & \sum_{i=1}^{M} \beta_{i j} \leq 1
\end{aligned}
$$

where $\lambda_{j} \in[0,1]$ and $1-\lambda_{j}$ are the weights of the $\mathcal{O}_{j}^{1}$ and $\widehat{O}_{j}^{2}$, respectively, which represent the significance of the userside and the network-side objectives on the decision making. When $\lambda_{j}=1$, the optimization problem (15) is converted to the user-side optimization, and to the network-side optimization when $\lambda_{j}=0$. Therefore, $\lambda_{j}$ should be determined by user's requirement and generally set to 0.5 considering the fairness between the user and the network. Considering that the objective function and constraints in (15) are linear and the number of available links for each user is limited, the multiobjective optimization can be solved by the integer programming method [25].

The link selection scheme we proposed on the user side is as shown in Figure 3. In general, the steps involved in this user-side scheme are summarized as follows.

Step 1. The prelink screening [17] is firstly performed to find candidate links that meet the requirements.

Step 2. If there is only one candidate link after the prelink screening, it will be chosen as the target link to establish connection. 


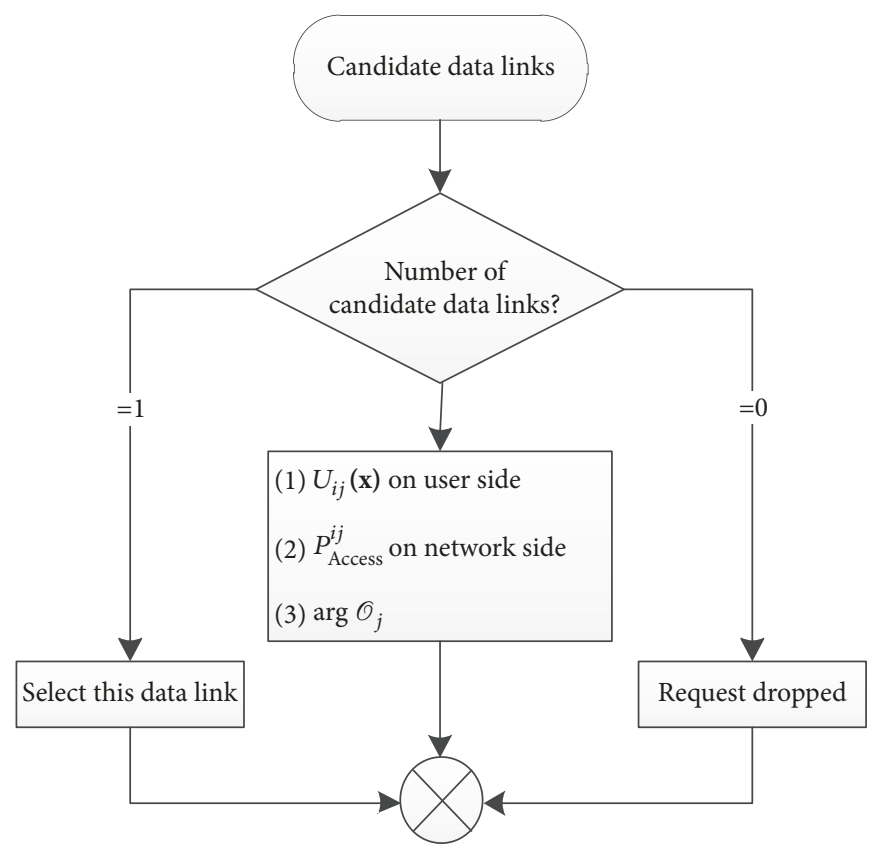

FIGURE 3: Link selection scheme on user side.

Step 3. If there is no available link, no connection will be established and the request will be dropped.

Step 4. If there are more than one candidate links, the link utility on the user side is first calculated, and then the access rate on the network side is calculated. Subsequently, the optimization problem $\mathcal{O}_{j}$ is constructed and solved, and the candidate link corresponding to the maximization of $\mathcal{O}_{j}$ is determined as the optimal link.

Due to the simple search method can be used to solve the optimization problem $\mathcal{O}_{j}$ because of the limited candidate links, we sort the links according to their $\mathcal{O}_{j}$ decreasingly to form a priority list, then the selected optimal link is the first element in this list.

3.2. Network-Assisted Selection Scheme. Due to the limited resources of heterogeneous aeronautical network, the target access network will have serious collisions if all selecting users in the same airspace utilize the above-mentioned user-side scheme to select the optimal link. In this multiuser access scenario, the heterogeneous aeronautical network needs to assist user to perform the link selection and ensure each user's access request with minimal modification.

In the overlapping airspace, both the selecting users and the nonselecting users are included among the $\mathrm{N}$ users. For the selecting user, it needs to select the optimal link according to the service requirement, whereas for the nonselecting user staying in certain link, it can be considered as a special selecting user who only has one candidate link that is actually the original link. We establish an optimization model for this scenario from the user's perspective to optimize all $\mathrm{N}$ users' requests as follows:

$$
\max \left\{\mathcal{O}_{1}, \mathcal{O}_{2}, \ldots, \mathcal{O}_{N}\right\}
$$

$$
\begin{array}{ll}
\text { s.t. } & \sum_{i=1}^{M} \beta_{i j} \leq 1 \\
& \sum_{j=1}^{N} \beta_{i j} \leq N_{\max }^{i}
\end{array}
$$

where $\sum_{j=1}^{N} \beta_{i j} \leq N_{\max }^{i}$ represents the number of users accessing the network $e_{i}$ cannot exceed the maximum number of users supported by this network.

The multiobjective optimization problem in (16) can be transformed into a maximum-minimum problem by the weighted Tchebycheff method [26] as follows:

$$
\begin{array}{ll}
\min & \max _{j}\left\{\phi_{j}\left(\mathcal{O}_{j}^{o}-\mathcal{O}_{j}\right)\right\} \\
\text { s.t. } & \phi_{j}>0 \\
& \sum_{i=1}^{M} \beta_{i j} \leq 1 \\
& \sum_{j=1}^{N} \beta_{i j} \leq N_{\max }^{i}
\end{array}
$$

where $\mathscr{O}_{j}^{o}=\max \mathscr{O}_{j}$ is the maximum of $\mathcal{O}_{j}$ and $\phi_{j}$ is the positive weight assigned to the user $j$ by the heterogeneous network.

The objective function in the optimization problem (17) can be further transformed into the following objective function:

$$
\min \sum_{j=1}^{N}\left\{\phi_{j}\left(\mathcal{O}_{j}^{o}-\mathcal{O}_{j}\right)\right\}
$$


Generally, considering the fairness between different users, we assume the same $\phi_{j}$ is reasonable for each user. Under this assumption, the solution of optimization problem (18) is a Pareto optimal solution for the multiobjective optimization problem [26]. As a combinatorial optimization, the complexity of optimization problem (18) increases exponentially with the number of users and their candidate links and will lead to a NP-hard problem which causes the traditional optimal search method searching one by one to be unimplementable.

In order to reduce the complexity of solving combinatorial optimization, suboptimal intelligent optimization algorithms, such as Genetic Algorithm (GA) [27] and Particle Swarm Optimization (PSO) [28], are generally used to approximate the optimal solution. However, the complexity of the intelligent optimization algorithm is still very high and the approximate optimum solution is not optimal for the users because the optimization problem (18) is established from the heterogeneous network's perspective.

Therefore, from the user's perspective, a priority distinction selection (PDS) algorithm on the network side is proposed by setting user's priority to maximize the number of users accessing their optimal links. We use the number of available links to distinguish the user priority, which means the smaller the number of available links, the higher the priority of the user. Particularly, the flexibility of the user decreases with the increasing number of available links. By setting the priority, the high priority users with low flexibility are allowed to access at first and followed by the low priority users subsequently. For the group of users with the same priority, the underload links can be accessed by the users who evaluate them as optimal links, and then the access of other users in this group will be allowed if the resources are plenty enough. Thereby, we can guarantee the maximal number of users to access their optimal links and the whole heterogeneous network in the meanwhile. The steps of the proposed PDS algorithm for optimization problem (18) are as shown in Algorithm 1.

Step 1. Firstly, the user $A_{j}^{i}$ with $i$ candidate links can be classified in the set $\mathbf{G}_{i}$; that means $A_{j}^{i} \in \mathbf{G}_{i}, 1 \leq i \leq M$. Correspondingly, the accessible network resources of $A_{j}^{i}$ are $\mathbf{R e}=\left\{\operatorname{Re}_{l}^{1}, \ldots, \operatorname{Re}_{t}^{i}\right\}, 1 \leq l, t \leq M$, where $\operatorname{Re}_{t}^{i}$ represents the remaining number of access user supported by $i$ th available link, which is sorted by user-side utility (note that the $\mathbf{R e}$ of each user is different).

Step 2. For a user with only a single available link $A_{j}^{1} \in \mathbf{G}_{1}$, the available resource of the link is $\mathbf{R e}=\left\{\operatorname{Re}_{l}^{1}\right\}$, and the link $e_{l}$ is the optimal access link. If $\operatorname{Re}_{l}^{1} \geq 1$, we can get $\beta_{l j}=1$ which means the user $A_{j}^{1}$ can access link $e_{l}$, and then Re should be updated after access; otherwise, the user $A_{j}^{1}$ cannot access any link because of $\beta_{l j}=0$.

Step 3. For users with multiple available links $A_{j}^{i} \in \mathbf{G}_{i}, i>$ 1 , the available resources are $\mathbf{R e}=\left\{\operatorname{Re}_{1}^{1}, \ldots, \operatorname{Re}_{t}^{i}\right\}$, where $l \neq t \& 1 \leq l, t \leq M$. Firstly, the user who can access the

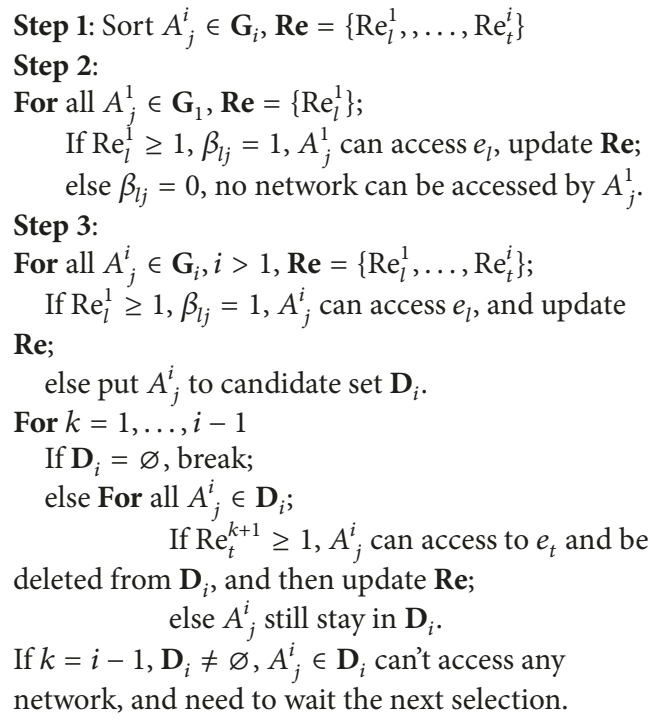

Algorithm 1: The proposed priority distinction selection algorithm.

optimal link is judged according to the link resources, which is the same as the user with single available link. For all users $A_{j}^{i} \in \mathbf{G}_{i}, i>1$, if $\operatorname{Re}_{l}^{1} \geq 1$, the user $A_{j}^{i}$ can access the optimal link $e_{l}$ because of $\beta_{l j}=1$, then Re is updated; however, for other users in $\mathbf{G}_{i}$, we sort them in a candidate set $\mathbf{D}_{i}$. For the users in the candidate set $A_{j}^{i} \in \mathbf{D}_{i}$, we repeat the above operation, and currently the user needs to search backwards for the suboptimal accessible link because of $\mathrm{Re}_{l}^{1}=0$. If the resources are available, the access is performed and the current user is deleted from $\mathbf{D}_{i}$; otherwise, the user continues to be retained in $\mathbf{D}_{i}$. Ultimately if there are still users in $\mathbf{D}_{i}$ after $i-1$ iterations, they cannot access to any link and need to wait for the next link selection.

Therefore, the proposed network-assisted selection scheme is based on the user's priority. First of all, the link selection mechanism on the user side is performed for each user, and then an access request with the sorted candidate link list is sent by the selected optimal link to heterogeneous aeronautical network. We assume that the heterogeneous network adopts a central control mode of SDN [19], the network receives the access requests of all users and then optimizes these requests according to (18) by PDS algorithm. Eventually, the network sends an acknowledgement to each user on the optimized access link, suppose that the user's reception detection is performed on each candidate link.

\section{Performance Evaluation}

Due to the poor availability and reliability of HF communications, HF links are not considered here. Assume that Iridium, VDL2, Inmarsat broadband global area network (BGAN) [29], and Mobile User Objective System (MUOS) [30] are the available links for aircraft in the overlapping airspace and 
TABLE 1: The specific attributes of different aeronautical links [17].

\begin{tabular}{|c|c|c|c|c|}
\hline \multirow{2}{*}{ Attributes } & \multicolumn{4}{|c|}{ Links } \\
\hline & Iridium & VDL2 & BGAN & MUOS \\
\hline \multirow{2}{*}{$\mathrm{BR}(\mathrm{kbps})$} & \multirow{2}{*}{ Up to 2.4} & \multirow{2}{*}{ Up to 31.5} & Class 4: up to 200 & \multirow{2}{*}{ Up to 64} \\
\hline & & & Class 6: up to 432 & \\
\hline $\mathrm{PD}(\mathrm{ms})$ & Up to 748 & Up to 2000 & Up to 1100 & Up to 1000 \\
\hline $\mathrm{RSS}(\mathrm{dBm})$ & - & - & - & - \\
\hline $\mathrm{CST}(\$ / \mathrm{MB})$ & 7 & 1 & 3 & 5 \\
\hline
\end{tabular}

TABLE 2: Utility values for different services.

\begin{tabular}{|c|c|c|c|c|c|c|c|c|}
\hline \multirow{3}{*}{ Links } & \multicolumn{4}{|c|}{ Attributes } & \multicolumn{4}{|c|}{ Services } \\
\hline & \multirow{2}{*}{ BR (kbps) } & \multirow{2}{*}{ PD (ms) } & \multirow{2}{*}{$\mathrm{RSS}(\mathrm{dBm})$} & \multirow{2}{*}{ CST (\$/MB) } & \multicolumn{2}{|c|}{$20 \mathrm{kbps}$} & \multicolumn{2}{|r|}{$2 \mathrm{kbps}$} \\
\hline & & & & & safety & non-safety & safety & non-safety \\
\hline Iridium & 2.1 & 100 & -84 & 7 & & & & 0.5260 \\
\hline VDL2 & 31.5 & 50 & -80 & 1 & - & 0.6163 & - & 0.6163 \\
\hline BGAN1 & 32 & 260 & -100 & 3 & & & & 0.1933 \\
\hline BGAN2 & 32 & 260 & -82 & 3 & & & & 0.1871 \\
\hline MUOS & 40 & 260 & -100 & 5 & & & & 0.3369 \\
\hline
\end{tabular}

their specific parameters are listed in Table 1 [17]. Since Class 4 and Class 6 of BGAN only differ in transmission rate, they can be expressed as BGAN1 and BGAN2, respectively. In this paper for so many attributes of each different link, only the corresponding bit rate (BR), packet delay (PD), received signal strength (RSS), and cost (CST) are considered.

4.1. The User-Side Selection Scheme. To begin with, we calculate the overall utility on the user side. Assume that the RSS vector of the five datalinks is $[-84,-80,-100,-82,-100]$ in $\mathrm{dBm}$ shown in Table 2 , the BR can be calculated by the current RSS accordingly. Since all three satellite links of BGAN1, BGAN2, and MUOS use synchronous orbit satellites, it can be assumed that the minimum propagation delay of one-hop is $260 \mathrm{~ms}$. And considering the cost of data transmission, we can see that the costs of Iridium and VDL2 are the highest and lowest compared with other links, respectively.

Take the 20kbps security service as an example to calculate the user-side utility. The prescreening of the available links is performed firstly. Since the VDL2 does not support the safety service, the available link sets are BGAN1, BGAN2, and MUOS as shown in Table 2. Under the principle of quality priority, the weights of attributes required by the service can be obtained by the i-TRUST method, where $\boldsymbol{\omega}=\left[\begin{array}{llll}0.30 & 0.15 & 0.50 & 0.05\end{array}\right]$. The overall utilities of 20kbps in different links calculated are $\mathrm{U}_{\mathrm{BGAN} 1}=0.1933, \mathrm{U}_{\mathrm{BGAN} 2}=$ 0.1871 , and $U_{\text {MUOS }}=0.3369$. For the 2 kbps security service, the other four links except VDL2 are candidate links. Under the same weights as the $20 \mathrm{kbps}$ service, the user-side utility of Iridium is 0.5260 . If only the link selection on the user side is considered, the best link for the $20 \mathrm{kbps}$ safety service is MUOS, and the best link for the $2 \mathrm{kbps}$ safety service is the Iridium link. For nonsafety services of $2 \mathrm{kbps}$ or $20 \mathrm{kbps}$, VDL2 is a candidate solution, but the Iridium link is not included in candidate set of the $20 \mathrm{kbps}$ nonsafety service.
According to Table 2, users with 20kbps or $2 \mathrm{kbps}$ nonsafety service tend to choose the most efficient VDL2 link.

Secondly, we calculate the access rate by the underload probability on the network side. We assume that there are a total of 200 aircraft in the overlapping airspace which remains stable at the current time, and the vector of the maximum number of users supported by the five datalinks is $[40,70,50,50,80]$. For different $\gamma_{i}$, the $P_{\text {Underload }}^{i}$ changing with the number of users in the target network is shown in Figure 4 . When the number of users in the Iridium network is less than $N_{\max }^{i}=40$, the $P_{\text {Underload }}^{i}$ of the Iridium network is all $100 \%$ that is because the resources of Iridium network occupy a tiny proportion of the total resources and the probability of other users choosing the Iridium is diminutive in the heterogeneous network. The underload probabilities of other networks increase with the ascending number of users due to the substantive scenarios of $N_{i 2 i}+N_{o 2 i}$. Considering more opportunities of current users switching to other links when the number of users held in the target network grows, the possible chances of users in other networks to handover to the target network increase, which augments the $P_{\text {Underload }}^{i}$ of the target network. Generally, $P_{\text {Underload }}^{i}$ increases with the descending $\gamma_{i}$ in Figure 4(b) compared with Figure 4(a), due to the decreasing $\gamma_{i}$ leading to the incremental availability of other users.

As for the access rate of different links shown in Figure 5, the change trend of $P_{\text {Access }}^{i j}$ is consistent with $P_{\text {Underload }}^{i}$ when $\alpha_{i j}$ remains unchanged, but the $P_{\text {Access }}^{i j}$ is lower than $P_{\text {Underload }}^{i}$. With the declining number of users in the target network, $P_{\text {Access }}^{i j}$ gradually decreases because so many resources can be utilized by other users with high selecting probabilities. On the contrary, a considerable $P_{\text {Access }}^{i j}$ can be achieved with the growing number of users benefiting from the more dynamic in the target network. 

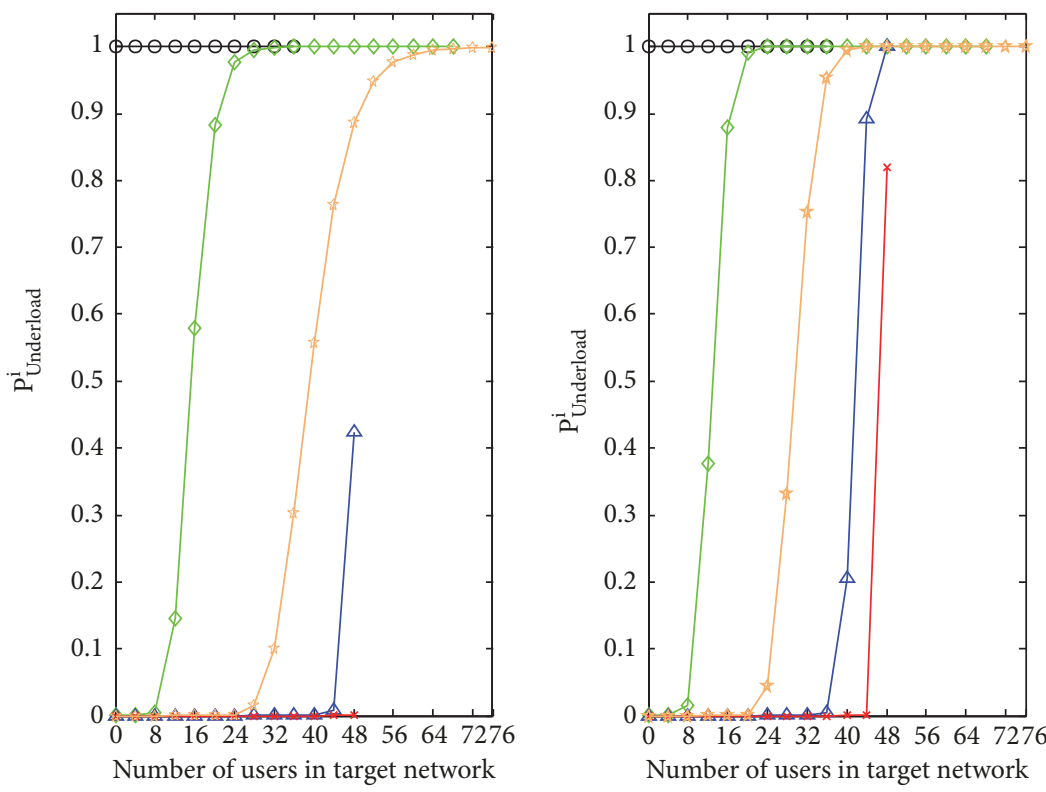

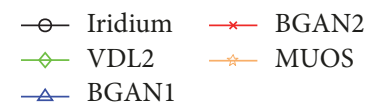

(a) $\gamma_{\mathrm{i}}=0.9$

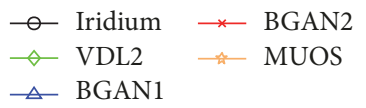

(b) $\gamma_{\mathrm{i}}=0.5$

FIgURE 4: The $P_{\text {Underload }}^{i}$ of the current network under different user numbers in different links.

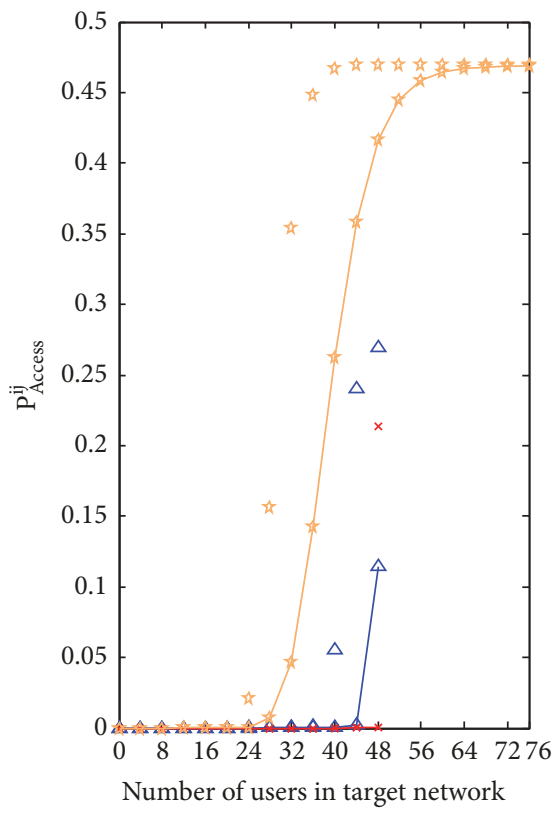

$\begin{array}{cl}\triangle & \text { BGAN1 } \gamma_{\mathrm{i}}=0.5 \\ \star & \text { BGAN2 } \gamma_{\mathrm{i}}=0.5 \\ \star & \text { MUOS } \gamma_{\mathrm{i}}=0.5 \\ \triangle & \text { BGAN1 } \gamma_{\mathrm{i}}=0.9 \\ \star & \text { BGAN2 } \gamma_{\mathrm{i}}=0.9 \\ \text { × } & \text { MUOS } \gamma_{\mathrm{i}}=0.9\end{array}$

(a) 20 kbps safety service
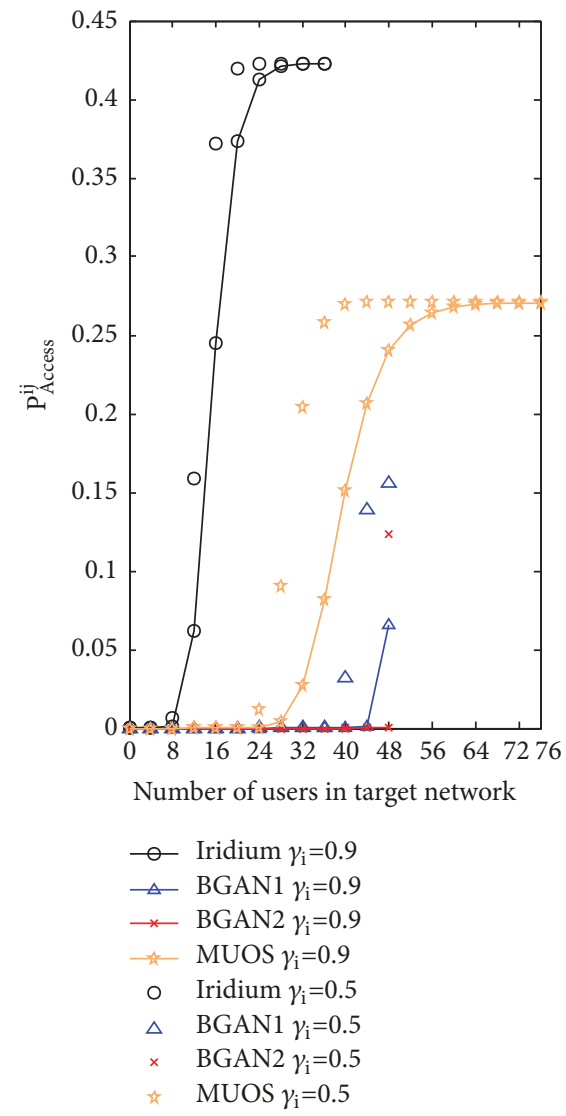

(b) 2 kbps safety service

FIgURE 5: The $P_{\text {Access }}^{i j}$ of $20 \mathrm{kbps}$ and $2 \mathrm{kbps}$ safety services. 


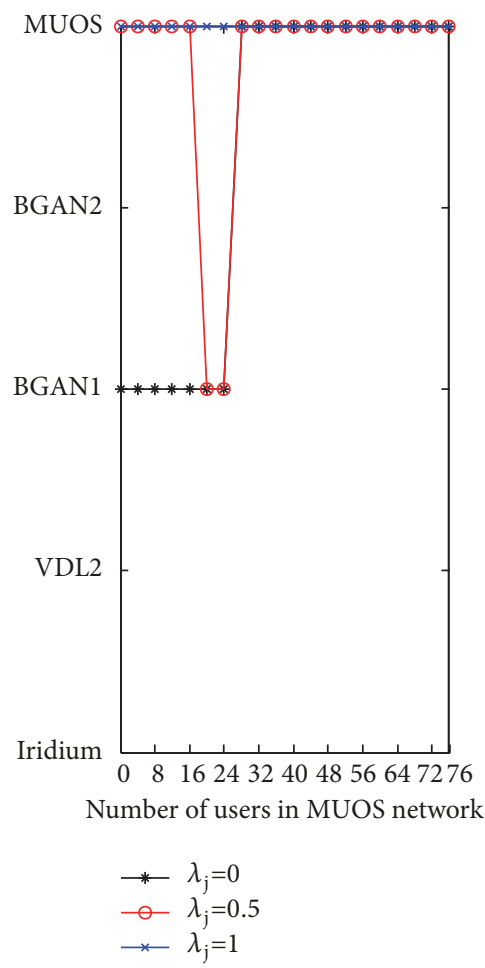

(a) 20 kbps safety service

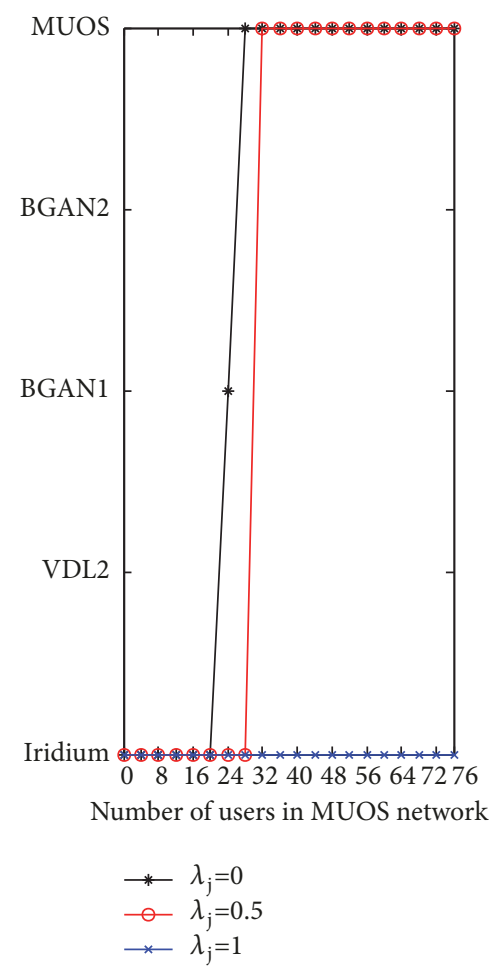

(b) 2 kbps safety service

FIGURE 6: The datalink selection results of different services in MUOS network.

TABLE 3: The available datalinks and their utilities of different users.

\begin{tabular}{|c|c|c|c|c|c|c|c|c|c|c|c|}
\hline \multirow{2}{*}{ User } & \multicolumn{5}{|c|}{ Network ID } & \multirow{2}{*}{ User } & \multicolumn{5}{|c|}{ Network ID } \\
\hline & 1 & 2 & 3 & 4 & 5 & & 1 & 2 & 3 & 4 & 5 \\
\hline 1 & 1 & 0 & 0 & 0 & 0 & 11 & 0 & 0.4 & 0.3 & 0.6 & 0 \\
\hline 2 & 0 & 1 & 0 & 0 & 0 & 12 & 0 & 0 & 0.5 & 0.3 & 0.4 \\
\hline 3 & 0 & 0 & 1 & 0 & 0 & 13 & 0.2 & 0 & 0.4 & 0 & 0.6 \\
\hline 4 & 0 & 0 & 0 & 0.5 & 0.7 & 14 & 0 & 0 & 0 & 0.4 & 0.3 \\
\hline 5 & 0.5 & 0.7 & 0 & 0 & 0 & 15 & 0.2 & 0 & 0.5 & 0 & 0.4 \\
\hline 6 & 0 & 0.4 & 0 & 0.6 & 0 & 16 & 0.2 & 0.4 & 0.5 & 0.3 & 0 \\
\hline 7 & 0 & 0 & 0.5 & 0 & 0.4 & 17 & 0 & 0.7 & 0.5 & 0.3 & 0.4 \\
\hline 8 & 0 & 0 & 0 & 0.4 & 0.6 & 18 & 0.2 & 0.7 & 0.5 & 0 & 0.4 \\
\hline 9 & 0.7 & 0 & 0 & 0.4 & 0 & 19 & 0.2 & 0.4 & 0.6 & 0.3 & 0.7 \\
\hline 10 & 0.2 & 0.6 & 0.5 & 0 & 0 & 20 & 0.7 & 0.8 & 0.6 & 0.3 & 0.4 \\
\hline
\end{tabular}

Finally, the results of user-side selection in MUOS network are shown in Figure 6 as an example. With the incremental number of users, the user selects the optimal link with the maximum $P_{\text {Access }}^{i j}$ when $\lambda_{j}=0$. Conversely, the user would like to select the link with maximum $U_{i j}(\mathbf{x})$ when $\lambda_{j}=1$, which results in conflictions among other users on the network side such as the Iridium link selected by $2 \mathrm{kbps}$ safety service in Figure 6(b). For $\lambda_{j}=0.5$, the user considers the over utility and access rate more fairly for the optimal link selection.

4.2. The Network-Assisted Selection Scheme. In order to simplify the presentation complexity, the scenario with the number of users $N=20$ and the number of above-mentioned links $M=5$ is considered, and the accessible networks corresponding to each user and their overall utilities are shown in Table 3. On the network side, we use Rate = $\left(\sum_{j=1}^{N} \max \mathscr{O}_{j}-\sum_{j=1}^{N} \mathcal{O}_{j}\right) / \sum_{j=1}^{N} \max \mathcal{O}_{j}$ to measure the performance of the proposed algorithm, where $\mathcal{O}_{j}$ is the value of each user accessing the corresponding network after using the proposed PDS algorithm. On the user side, we use the number of users accessing the heterogeneous network $N_{a}$ and the number of users accessing optimal link $N_{o}$ to measure the performance. The proposed PDS algorithm is compared with the GA and PSO intelligent algorithms, whose number 


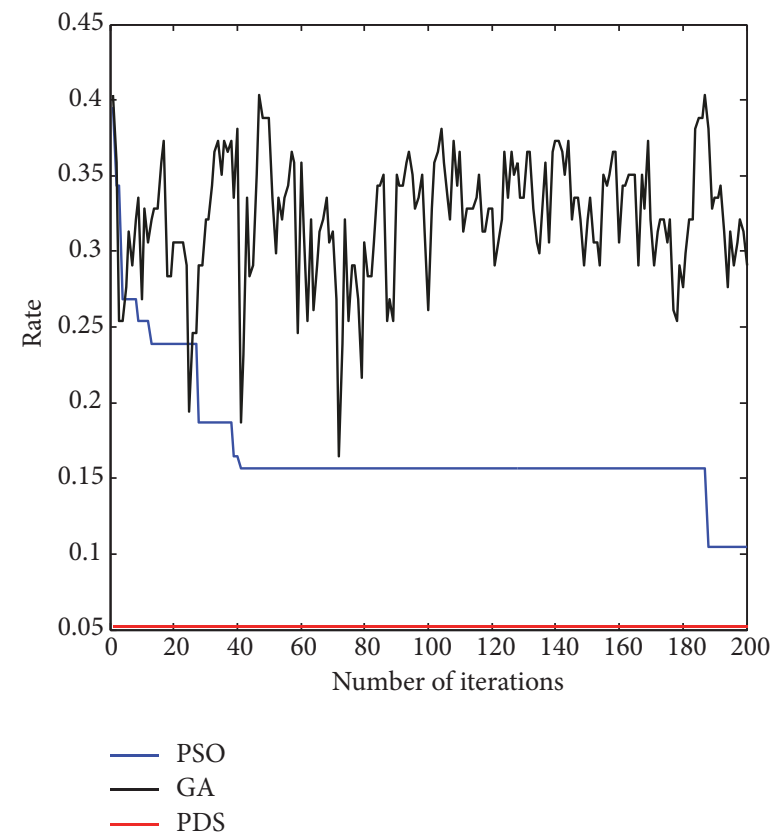

Figure 7: The Rate of different algorithms under Renet = $\left[\begin{array}{lllll}3 & 5 & 4 & 3 & 5\end{array}\right]$.

of iterations is set to 200 and population size is 100 for saving the processing time.

Firstly, 20 network resources are taken as an example to verify the performance of proposed algorithm. We assume that the number of available resources corresponding to each network is Renet $=\left[\begin{array}{lllll}3 & 5 & 4 & 3 & 5\end{array}\right]$. Without considering resource constraints, the maximum cumulative utility achieved by the access link is 13.4 according to Table 3. As shown in Figure 7 , the maximum cumulative utility obtained by the GA algorithm is 11.2, and correspondingly the minimum of Rate is $16.42 \%$ in the 200 iterations. Simultaneously when Rate reaches its minimum, Figure 8 presents that $N_{a}$ is equal to 19 and $N_{o}$ equals 9, which means 10 users access the nonoptimal links and one user cannot access the heterogeneous network. Through multiple selection, crossover and mutation operations, the Rate of GA algorithm does not converge to a stable value, because the above operations cause individuals to become random and do not evolve in an optimal direction during evolution. Moreover, for all access schemes corresponding to individuals in GA algorithm, not all of them meet resource constraints. On the contrary, the PSO algorithm gradually converges to the local optimal solution through multiple iterations. The maximum utility value obtained at this time is 12 , and the Rate gradually stabilizes at $10.45 \%$. Meanwhile, $N_{a}=20$ and $N_{o}=12$, which means there are 8 users switching from the expected optimal link to the nonoptimal link, so the PSO performance is better than the GA algorithm. From Figure 7 we can see that the PDS algorithm has the best performance, because the PDS algorithm is a deterministic search algorithm which does not need multiple iterations and has low complexity. The cumulative utility obtained by PDS algorithm is 12.7 , and the Rate is only $5.22 \%$. Currently, the $N_{a}$ is equal to 20 and $N_{o}$

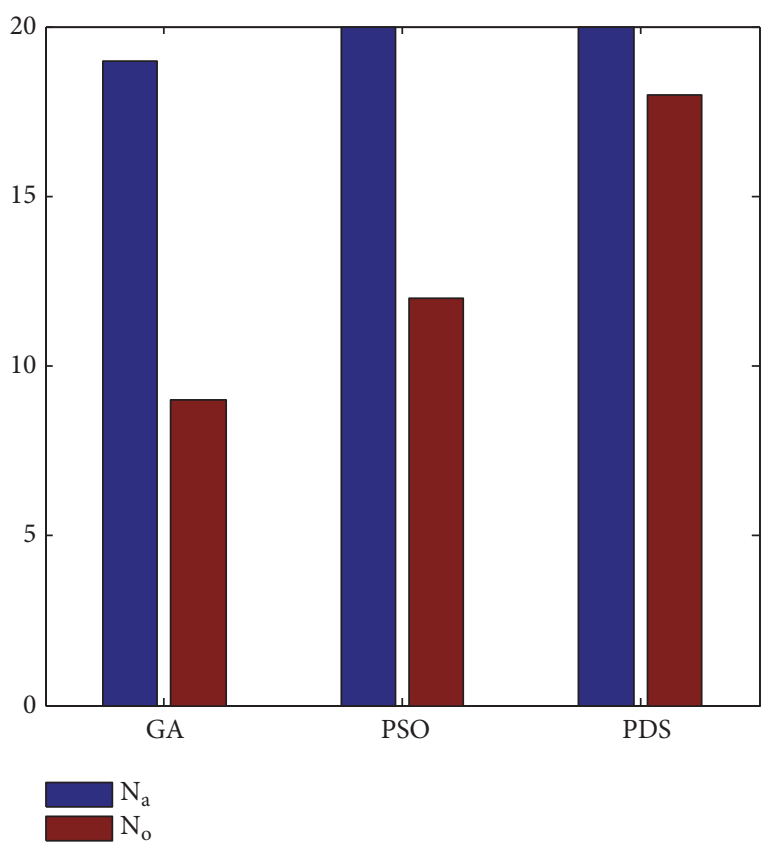

Figure 8: $N_{a}$ and $N_{o}$ of different algorithms under Renet = $\left[\begin{array}{lllll}3 & 5 & 4 & 3 & 5\end{array}\right]$.

equals 18 according to Figure 8, which means only the 19th and the 20th users cannot access the optimal link. Therefore, the required modification for users' link selection in this PDS algorithm is the smallest compared with GA and PSO.

When the resources of heterogeneous network are less than the number of users, for example, in the case where the number of access network resources is Renet $=\left[\begin{array}{lllll}2 & 4 & 3 & 2 & 4\end{array}\right]$, the three algorithms are evaluated. As can be seen from Figure 9, the GA algorithm still does not converge with the reduction of network resources, and the minimum of Rate is $33.58 \%$ in 200 iterations. Meanwhile $N_{a}=14$ and $N_{o}=$ 10 can be reached by GA algorithm according to Figure 10, which means one user cannot access and four users have to access to nonoptimal links. With the reduction of resources, the PSO algorithm gradually improves its search performance and is superior to the PDS algorithm in the Rate, which is stable at $4.67 \%$ as the iteration increases, because the search range of PSO is drastically reduced and it can reach the optimal solution from the perspective of the network. Only $N_{o}=12$ out of $N_{a}=15$ users can access to their optimal links when using PSO algorithm; however, for the PDS algorithm the $N_{o}$ can reach 13 with $N_{a}=15$ although the Rate is $27.61 \%$ at this time. From the user's perspective when the network resources are gradually reduced, the PDS algorithm is still better than PSO algorithm and can reduce the complexity without the multiple iterations. Therefore, the DSP algorithm can maximize the number of users accessing the optimal links and minimize the user' modification greatly compared with GA and PSO algorithms.

\section{Conclusions}

This paper proposes a novel network-assisted datalink selection mechanism for heterogeneous aeronautical network in 


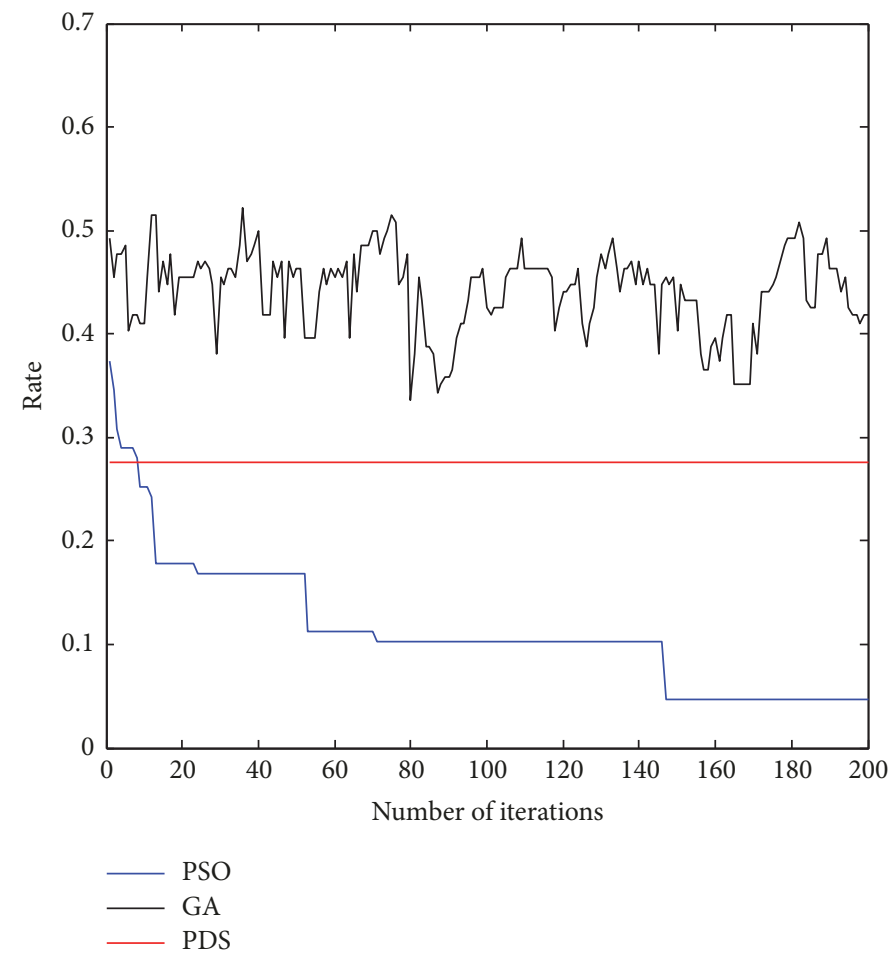

FIGURE 9: The Rate of different algorithms under Renet $=\left[\begin{array}{lllll}2 & 4 & 3 & 2 & 4\end{array}\right]$.

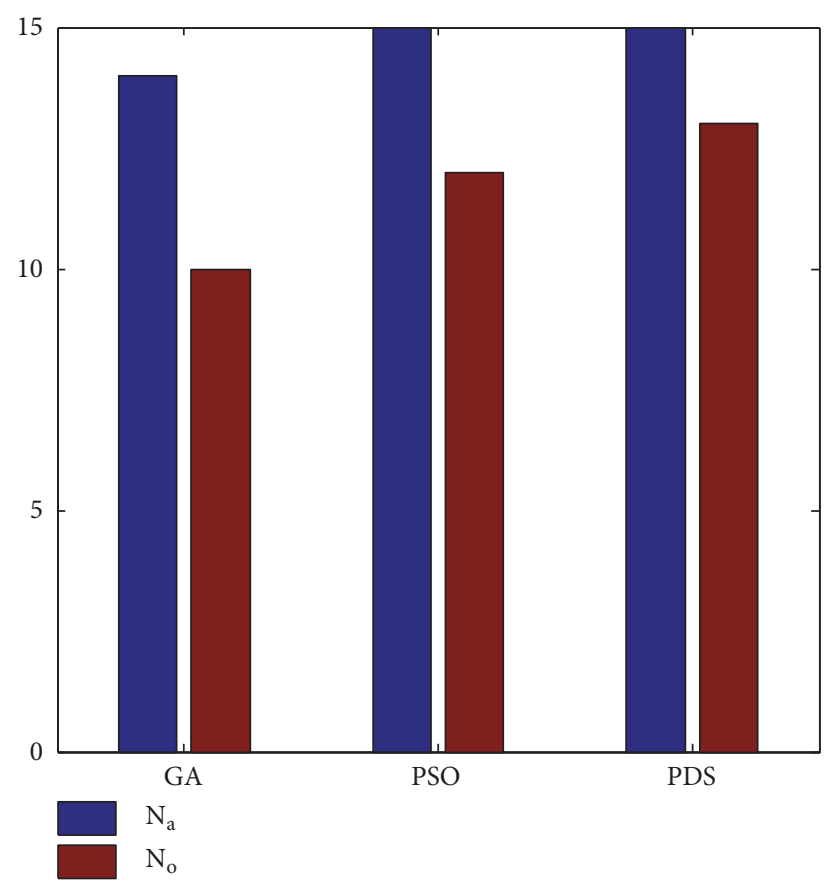

Figure 10: $N_{a}$ and $N_{o}$ of different algorithms under Renet = $\left[\begin{array}{lllll}2 & 4 & 3 & 2 & 4\end{array}\right]$.

multilink overlapping airspace. For a single-user selection, the user combines the user-side utility with the networkside access rate to optimize and select the optimal link. For multiuser selection, combined with the network-side central control mode, a priority distinction selection algorithm with low complexity is proposed to assist users to perform link selection, maximize the number of users accessing their optimal links, and minimize the user-side modification. The effectiveness of the proposed selection scheme with proposed algorithm is validated by simulations. In the future, we will study the multilink selection, especially dual-connectivity scheme, to support the high mobility of aircraft. By utilizing the multilink connectivity, the aircraft can reduce the unnecessary handover for realizing a seamless communication and make use of the diversity to improve the robustness and reliability. Furthermore, under the constraint of limited resources we will optimize the global performance of the aeronautical heterogeneous network considering dual-connectivity users in the overlapping airspace.

\section{Data Availability}

The data used to support the findings of this study is included within the article.

\section{Conflicts of Interest}

The authors declare that there are no conflicts of interest regarding the publication of this paper.

\section{Acknowledgments}

This work was supported by the National Natural Science Foundation of China (Grant 61701521), the China Postdoctoral Science Foundation (Grant 2016M603044), and 
the Shaanxi Provincial Natural Science Foundation (Grant 2018JQ6074).

\section{References}

[1] AIRBUS GLOBAL MARKET FORECAST 2017-2036. http://www .aircraft.airbus.com/market/global-market-forecast20172036/? eID=maglisting_push\&amp;tx_maglisting_pi1\%5BdocID\%5D= 233685.

[2] D. G. Depoorter and W. Kellerer, "Designing the Air-Ground Data Links for Future Air Traffic Control Communications," IEEE Transactions on Aerospace and Electronic Systems, 2018.

[3] I. Jawhar, N. Mohamed, J. Al-Jaroodi, D. P. Agrawal, and S. Zhang, "Communication and networking of UAV-based systems: Classification and associated architectures," Journal of Network and Computer Applications, vol. 84, pp. 93-108, 2017.

[4] X. Wang, Z. Ouyang, H. Song, and Q. Lin, "Collaborative unmanned aerial systems for effective and efficient airborne surveillance," in Proc. SPIE 10652, Disruptive Technologies in Information Sciences, vol. 106520F, 2018.

[5] X. Yue, Y. Liu, J. Wang, H. Song, and H. Cao, "Software Defined Radio and Wireless Acoustic Networking for Amateur Drone Surveillance," IEEE Communications Magazine, vol. 56, no. 4, pp. 90-97, 2018.

[6] J. Fan, D. Li, R. Li, T. Yang, and Q. Wang, "Analysis for cooperative combat system of manned-unmanned aerial vehicles and combat simulation," in Proceedings of the in IEEE International Conference on Unmanned Systems (ICUS), IEEE, pp. 204-209, 2017.

[7] B. Kubera and M. Ehammer, "A survey of multilink concepts for aeronautical data link communications," in Proceedings of the 2013 13th Annual Integrated Communications, Navigation and Surveillance Conference, ICNS 2013, USA, April 2013.

[8] Next generation air transportation system - Integrated plan, U.S. Dept. Transp., Washington, DC, 2004.

[9] State of progress with the project to implement the new generation European Air Traffic Management System (SESAR), European Union, Brussels, Belgium, 2007.

[10] M. Schnell and S. Scalise, "NEWSKY - concept for NEtworking the SKY for civil aeronautical communications," IEEE Aerospace and Electronic Systems Magazine, vol. 22, no. 5, pp. 25-29, 2007.

[11] S. Plass, "Seamless networking for aeronautical communications: One major aspect of the SANDRA concept," IEEE Aerospace and Electronic Systems Magazine, vol. 27, no. 9, pp. 21-27, 2012.

[12] M. Schnell, U. Epple, D. Shutin, and N. Schneckenburger, "LDACS: Future aeronautical communications for air-traffic management," IEEE Communications Magazine, vol. 52, no. 5, pp. 104-110, 2014.

[13] G. Bartoli, R. Fantacci, and D. Marabissi, "AeroMACS: A new perspective for mobile airport communications and services," IEEE Wireless Communications Magazine, vol. 20, no. 6, pp. 4450, 2013.

[14] VHF air-ground digital link (VDL) mode 2, Technical characteristics and methods of measurement for ground-based equipment, Part 1: Physical layer and MAC sub-layer, ETSI, Sophia Antipolis, France, 2010, pp. 1-53.

[15] C. Kissling, "Data link selection in mobile aeronautical telecommunication networks," in Proceedings of the 27th IET and AIAA International Communications Satellite Systems Conference (ICSSC 2009), pp. 1-8, Edinburgh, UK.
[16] L. Wang and D. Binet, "TRUST: A trigger-based automatic subjective weighting method for network selection," in Proceedings of the 2009 5th Advanced International Conference on Telecommunications, AICT 2009, pp. 362-368, Italy, May 2009.

[17] A. S. Alam, Y.-F. Hu, P. Pillai, K. Xu, and J. Baddoo, "Optimal Datalink Selection for Future Aeronautical Telecommunication Networks," IEEE Transactions on Aerospace and Electronic Systems, vol. 53, no. 5, pp. 2502-2515, 2017.

[18] P. W. Lemme, S. M. Glenister, and A. W. Miller, "Iridium aeronautical satellite communications," IEEE Aerospace and Electronic Systems Magazine, vol. 14, no. 11, pp. 11-16, 1999.

[19] L. Qiang, J. Li, Y. Ji, and C. Huang, "A novel software-defined networking approach for vertical handoff in heterogeneous wireless networks," Wireless Communications and Mobile Computing, vol. 16, no. 15, pp. 2374-2389, 2016.

[20] D. Jiang, L. Huo, Z. Lv, H. Song, and W. Qin, "A Joint MultiCriteria Utility-Based Network Selection Approach for Vehicleto-Infrastructure Networking," IEEE Transactions on Intelligent Transportation Systems, vol. 19, no. 10, pp. 3305-3319, 2018.

[21] L. Qiang, J. Li, and E. Altman, "A novel distributed network selection scheme for heterogeneous wireless network environments," IEEE Transactions on Control of Network Systems, vol. 4, no. 3, pp. 575-586, 2017.

[22] J. Kellokoski, J. Koskinen, and T. Hämäläinen, "Always best connected heterogeneous network concept," Wireless Personal Communications, vol. 75, no. 1, pp. 63-80, 2014.

[23] M. Bin, D. Hong, and X. Xianzhong, "Two-sided Matching Model Based Vertical Handover Algorithm in Heterogeneous Wireless Networks," Journal of Electronics Information Technology, vol. 40, no. 2, pp. 421-429, 2018.

[24] L. A. Zadeh, "Optimality and non-scalar-valued performance criteria," IEEE Transactions on Automatic Control, vol. 8, no. 1, pp. 59-60, 1963.

[25] J. S. Borrero, C. Gillen, and O. A. Prokopyev, "A simple technique to improve linearized reformulations of fractional (hyperbolic) 0-1 programming problems," Operations Research Letters, vol. 44, no. 4, pp. 479-486, 2016.

[26] G. Yu, Y. Jiang, L. Xu, and G. Y. Li, "Multi-objective energyefficient resource allocation for multi-RAT heterogeneous networks," IEEE Journal on Selected Areas in Communications, vol. 33, no. 10, pp. 2118-2127, 2015.

[27] D. Whitley, "A genetic algorithm tutorial," Statistics and Computing, vol. 4, no. 2, pp. 65-85, 1994.

[28] I. C. Trelea, "The particle swarm optimization algorithm: convergence analysis and parameter selection," Information Processing Letters, vol. 85, no. 6, pp. 317-325, 2003.

[29] A. Franchi, A. Howell, and J. Sengupta, "Broadband mobile via satellite Inmarsat BGAN," in Proceedings of the IEE Seminar on Broadband Satellite: The Critical Success Factors Technology, Services and Markets, pp. 23-23, London, UK, 2002.

[30] J. D. Oetting and T. Jen, "The mobile user objective system," Johns Hopkins APL Technical Digest, vol. 30, no. 2, pp. 103-112, 2011. 


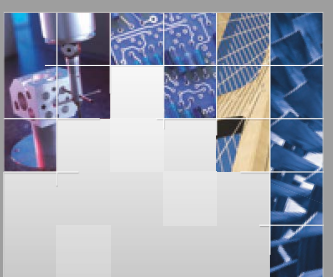

\section{Enfincering}
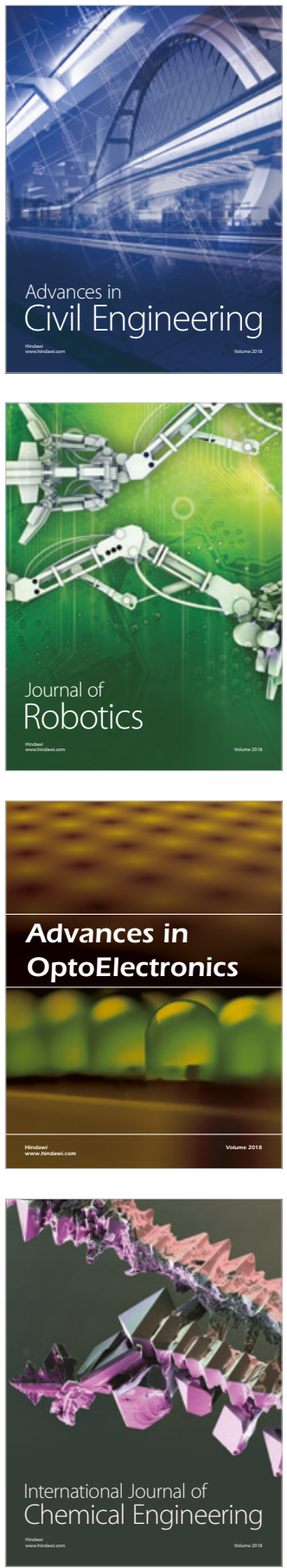

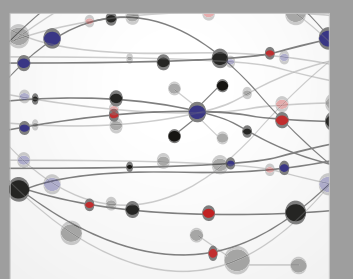

\section{Rotating \\ Machinery}

The Scientific World Journal

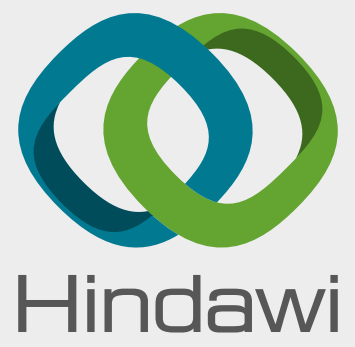

Submit your manuscripts at

www.hindawi.com
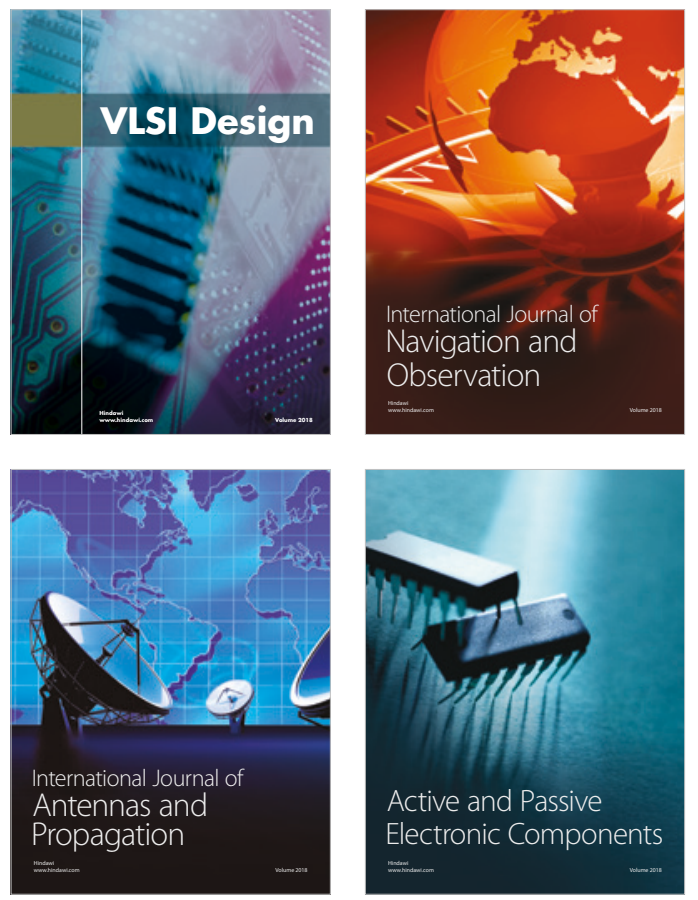
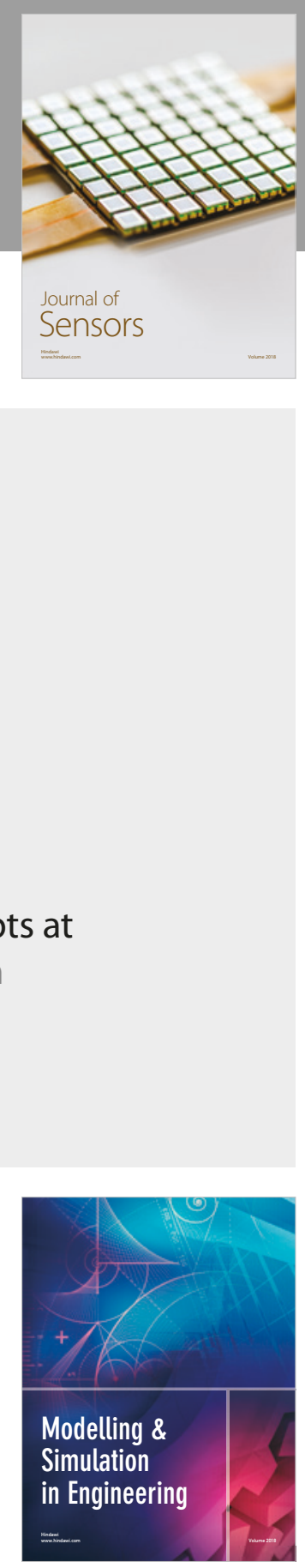

\section{Advances \\ Multimedia}
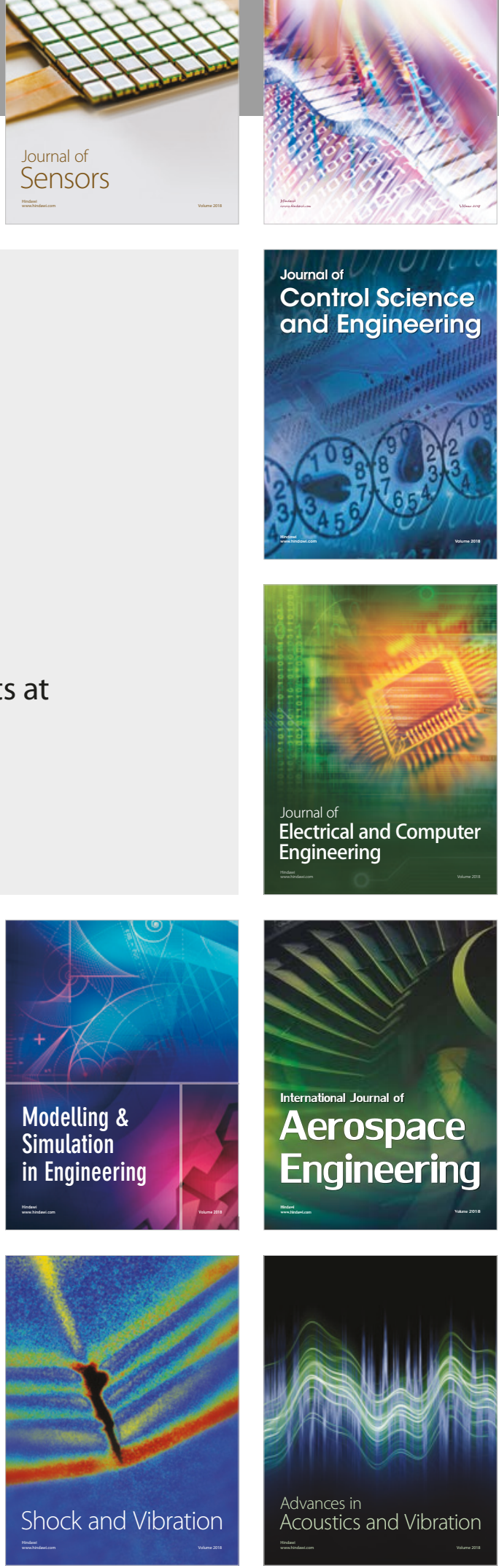